\title{
Synchronic and diachronic identity for elementary particles
}

\section{Tomasz Bigaj $^{1}$}

Received: 24 January 2020 / Accepted: 18 June 2020 / Published online: 14 July 2020

(C) The Author(s) 2020

\begin{abstract}
The main focus of this paper is on the notion of transtemporal (diachronic) identity applied to quantum particles. I pose the question of how the symmetrization postulate with respect to instantaneous states of particles of the same type affects the possibility of identifying interacting particles before and after their interaction. The answer to this question turns out to be contingent upon the choice between two available conceptions of synchronic individuation of quantum particles that I call the orthodox and heterodox approaches. I argue that the heterodox approach offers a better explanation of the known experimental facts regarding particle interactions, and I probe deeper the concepts of synchronic and diachronic identity emerging from this approach.
\end{abstract}

Keywords Diachronic identity · Synchronic identity · Individuation · Quantum particles · Permutation invariance $\cdot$ Scattering experiments $\cdot$ Interference effects $\cdot$ Transition amplitudes

\section{Introduction}

The problem of identity and individuality (discernibility, reference, etc.) in nonrelativistic quantum mechanics is typically considered in the context of instantaneous states of many particles of the same type. Given that at any moment the state of a system of such particles has to possess certain symmetry properties (it has to be either symmetric or antisymmetric), we may ask the question of how this fact affects the possibility of discerning the individual particles within the system, making reference to a selected subset of them, making sure that they are numerically distinct, and so forth. When answering these questions, we take a look at the momentary state of the involved particles, as if taking a snapshot of their history. We may call this perspective

Tomasz Bigaj

t.f.bigaj@uw.edu.pl; tfbigaj@gmail.com

1 Institute of Philosophy, University of Warsaw, ul. Krakowskie Przedmiescie 3, 00-047 Warsaw, Poland 
"synchronic", as opposed to the diachronic perspective which compares states and properties of selected objects across different temporary instants. The issue of the diachronic identity and identification of quantum particles has received relatively little attention in the literature, apart from some general remarks regarding the non-existence of well-defined trajectories in quantum mechanics and the ensuing difficulty with tracing the temporal evolution of quantum objects. ${ }^{1}$ The current paper hopes to fill this gap.

The terminological distinction between diachronic identity and synchronic identity may suggest, on a superficial reading, that we are dealing here with two distinct genera of identity relations. Indeed, some philosophers suggest that only synchronic identity is proper numerical identity, whereas diachronic identity is a different, albeit connected relation that applies to objects persisting in time. ${ }^{2}$ One possible argument in favor of this approach is based on the fact that temporal things clearly change over time: at one time $t_{1}$ an object can possess property $P$, while at a different time $t_{2}$ it can lose it. If diachronic identity was just ordinary numerical identity, the argument goes, we would have a violation of the logical Leibniz law. To stave off this unfortunate consequence, some insist that diachronic identity is a relation that connects numerically distinct entities, namely temporal slices of a four-dimensional whole that constitutes an object persisting in time. In my current analysis I will adopt an alternative approach, according to which both synchronic and diachronic identities are numerical identities. The only difference between the two 'types' of identity lies in the way we pick out appropriate objects whose numerical identity/distinctness we are evaluating. ${ }^{3} \mathrm{We}$ consider the problem of synchronic identity each time we describe objects by referring to their properties or relations (or states) taken at the same time. If, on the other hand, we use descriptions taken at different moments, we ask questions about diachronic identity, which nevertheless are still questions about whether the thing described at $t_{1}$ is the same entity as the thing described at $t_{2}$. What we relativize to time is descriptions by which we refer to objects, and not the relation of identity, which is in some sense atemporal.

It is to be expected that the symmetrization postulate, which imposes restrictions on the mathematical form of the instantaneous states of groups of particles, should first and foremost have an effect on the statements regarding the synchronic identity and identification of particles. Broadly speaking, there are two options here to choose from. First, there is the orthodox approach to quantum identity and individuality, which insists that particles of the same type (i.e. possessing the same state-independent properties, such as charge, rest mass, total spin and so on) that form a system described by a symmetric/antisymmetric function can never differ with respect to their quantum-

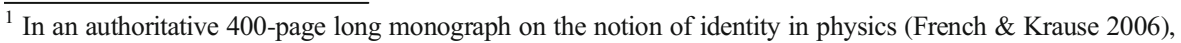
the authors devote less than two pages (pp. 19-20) to a brief characterization of the notion of transtemporal identity, followed by even more brief remarks regarding the impossibility of reidentification of quantum particles due to the failure of spatiotemporal continuity (pp. 122-123). The problem of the transtemporal identity of quantum particles is treated slightly more comprehensively in (Saunders 2009), where the author discusses the connection between the types of symmetries (fermionic and bosonic) and the transition amplitudes in scattering experiments of the kind we will consider later in this article.

${ }^{2}$ See (Galois 2016) for an overview of this problem.

${ }^{3}$ The problem with the Leibniz law on this approach is usually solved by relativizing properties to temporal moments, so that there is no longer a logical contradiction between, let's say, a poker's being hot at time $t_{1}$ and being cold at time $t_{2}$. For a deeper analysis of what it means to relativize a property to time, see e.g. (Haslanger 2003).
} 
mechanical properties. ${ }^{4}$ This is supposed to be a straightforward consequence of the permutation-invariance of the composite state they participate in, coupled together with the standard recipe of how to calculate the so-called reduced states of the components of a composite system. It is a simple mathematical fact that the partial traces of the density matrix representing the state of the whole system taken over all but one particle are identical no matter which particle we choose, for all symmetric/antisymmetric states. And because these partial traces represent the reduced states of individual particles, the case seems to be closed: no particles of the same type are discernible by their properties (in standard terminology: they are not absolutely discernible ${ }^{5}$ ). Thus quantum objects violate the venerated Principle of the Identity of Indiscernibles (stating that no two objects can possess the exact same properties).

An alternative, heterodox approach, which is steadily gaining popularity, questions the Indiscernibility Thesis, that is the claim that particles of the same type are never discernible by their properties. ${ }^{6}$ The way to do that is by denying that the labels used to differentiate between the copies of identical Hilbert spaces in the N-fold tensor product also refer to individual particles. Instead, individuation should be done with the help of symmetric projectors that represent disjunctive properties of the sort "particle 1 has property $\mathrm{P}$ and particle 2 has property $\mathrm{Q}$ or particle 1 has property $\mathrm{Q}$ and particle 2 has property P". 7 According to this new approach, groups of 'identical' fermions can be sometimes treated as if they momentarily occupied separable states. That is, if the state of a system of fermions can be obtained as a result of antisymmetrizing a product of orthogonal one-particle states, individual fermions can be ascribed properties corresponding to these one-particle states. This ascription, however, does not connect discerning properties with individual labels in the tensor product, since - as we pointed out - labels no longer play the referential role. Thus we can say for instance that one particle possesses spin up in a given direction and another particle possesses spin down in the same direction, but not that particle 1 possesses spin up while particle 2 possesses spin down.

I will not discuss here in detail the arguments in the debate between the orthodox and heterodox approaches to the problem of the individuation of quantum particles

\footnotetext{
${ }^{4}$ Virtually all works on the issue of identity and indistinguishability in quantum mechanics published before 2010 subscribe to the view I call orthodox. Typical examples of this approach that place particular emphasis on the purported indiscernibility of same-type quantum particles by properties are (French \& Redhead 1988), (Redhead \& Teller 1992), (Butterfield 1993), (Huggett 2003), (French \& Rickles 2003), (French \& Krause 2006), (Muller \& Saunders 2008), (Huggett \& Norton 2014).

${ }^{5} \mathrm{I}$ am refraining from using the more natural term "absolute indiscernibility" for fear of confusion. The adjective "absolute" indicates the relative strength of the relation to which this adjective is attributed. Thus, absolute discernibility is the strongest of all three grades of discernibility discussed in the literature (the remaining ones being relative and weak discernibility). However, by contraposition, the negation (complement) of absolute discernibility is the weakest of the three! By using the term "absolute indiscernibility" I might give the wrong impression that I have in mind the negation of weak discernibility (sometimes referred to as "utter indiscernibility"). See a discussion of this small but not insignificant terminological problem in (Ladyman et al. 2012, p. 165).

${ }^{6}$ The heterodox approach to quantum individuation has its origins in an extensive analysis of the concept of entanglement of indistinguishable particles given in (Ghirardi et al. 2002). Its most complete exposition can be found in (Caulton 2014, 2015). Similar ideas have been endorsed in (Dieks \& Lubberdink 2011, 2020), (Saunders 2013, 2015), (Friebe 2014) and (Bigaj 2015, 2016, 2020a, 2020b).

${ }^{7}$ In the quantum-mechanical formalism properties of this kind are represented by symmetric projection operators of the form $P \otimes Q+Q \otimes P$, where $P$ and $Q$ are orthogonal projectors acting in one-particle Hilbert spaces.
} 
(however, this topic will be briefly touched upon in the concluding remarks of the paper). Instead, I will proceed straight away to the central topic of the paper which is how the permutation-invariance of states impacts the identification of quantum systems over time. Typical situations in which we may ask questions regarding the diachronic identity of quantum objects are experiments involving interactions between particles, such as scattering processes. When two particles collide and then 'bounce off' each other, the issue of their pre- and post-interaction identifications arises. That is, we consider the question of whether the particle detected at a particular location after the interaction is identical with the particle coming 'from the left' or with the one coming 'from the right'. In order to address this question more precisely, we have to resort to the standard quantum-mechanical formalism which calculates the probabilities of finding particles scattered in given directions using so-called transition amplitudes.

\section{The case of distinguishable particles}

Let us consider first the case of two distinguishable particles possessing the same electric charge, for instance a proton and a positron. Suppose that the proton is coming from the left while the positron is arriving from the right (see Fig. 1). The incident wave function of the proton at the initial moment $t_{0}$ can be written as $\left|p^{+}, \psi_{L}\right\rangle$, while the positron is characterized by the wave function coming from the right $\left|e^{+}, \psi_{R}\right\rangle{ }^{8}$ Because we are dealing with distinguishable particles, the joint state of the entire proton-positron system at $t_{0}$ will be just the simple product.

$$
\left|p^{+} e^{+}, t_{0}\right\rangle=\left|p^{+}, \psi_{L}\right\rangle_{1} \otimes\left|e^{+}, \psi_{R}\right\rangle_{2}
$$

of two one-particle states. Here labels 1 and 2 refer unambiguously to separate particles thanks to the fact that these particles can be distinguished by their state-independent properties (for instance rest mass). The interaction between the particles can be most generally presented with the help of the unitary evolution operator $U(t)$, where $t=t_{1}-t_{0}$ (the evolution operator is a function of the Hamiltonian $H: U(t)=e^{-\frac{i t H}{h}}$ ). Acting with this operator on the initial state we can calculate the state of the entire system at any time $t_{1}>t_{0}$ as follows:

$$
\left|p^{+} e^{+}, t_{1}\right\rangle=U\left(t_{1}-t_{0}\right)\left|p^{+}, \psi_{L}\right\rangle_{1} \otimes\left|e^{+}, \psi_{R}\right\rangle_{2}
$$

If the interaction between the proton and the positron is assumed to happen only within a certain small radius, the scattered wave function $\left|p^{+} e^{+}, t_{1}\right\rangle$ of both particles can be approximated as the product of two wave functions that propagate spherically in all directions from the point of collision (see Sakurai \& Napolitano 2011, p. 396). Thus for each direction in space there will be a non-zero probability to register a particle

\footnotetext{
${ }^{8}$ Wave functions (packets) $\psi_{L}$ and $\psi_{R}$ represent states of particles in motion with opposite momenta $\boldsymbol{p}$ and $-\boldsymbol{p}$ along the line connecting $L$ and $R$. The cases used in this and subsequent sections are modeled on (CohenTannoudji et al. 1978, pp. 1403-1408). See also (Feynman et al. 1965, chaps. 3 and 4).
} 

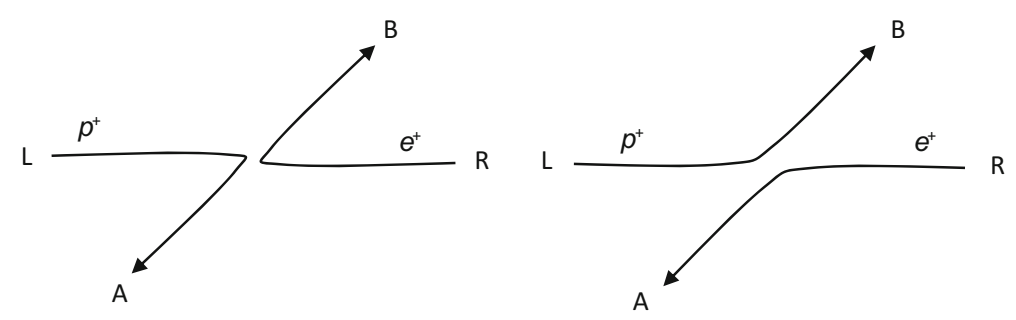

Fig. 1 Two possible trajectories in a scattering experiment

scattered in that direction. Suppose now that we are interested in calculating the probability that after the interaction the particles will be found at a particular selected angle (in the center-of-mass reference frame). In order to find this probability, we have to take first the evolved state $\left|p^{+} e^{+}, t_{1}\right\rangle$ of the entire proton-positron system at $t_{1}$ and then calculate, using the Born rule, the probability that the measurement will find the system in a particular final state $\left|p^{+} e^{+}, \psi_{f}\right\rangle$, where $\psi_{f}$ describes the wave function of both particles propagating alongside the selected direction ( $A B$ on Fig. 1). However, given that the outcome (scattering at a certain angle) can be realized in two different and physically distinguishable ways, we have to calculate the appropriate probabilities separately for each physical realization.

If $\left|p^{+}, \psi_{A}\right\rangle$ (resp. $\left.\left|p^{+}, \psi_{B}\right\rangle\right)$ represents the wave function of the proton registered as scattered in the $A$ (resp. $B$ ) direction, with an analogous interpretation of kets $\left|e^{+}, \psi_{A}\right\rangle$, $\left|e^{+}, \psi_{B}\right\rangle$, then the two possible final states can be written as $\left|p^{+}, \psi_{A}\right\rangle_{1} \otimes\left|e^{+}, \psi_{B}\right\rangle_{2}$ and $\left|p^{+}, \psi_{B}\right\rangle_{1} \otimes\left|e^{+}, \psi_{A}\right\rangle_{2}$. The first state corresponds to the situation when the proton ends up being scattered in the $A$ direction and the positron in the $B$ direction, while the second state has the particles fly off in alternated directions. And in order to calculate the probability that the system will be found in a specific state $\left|p^{+} e^{+}, \psi_{f}\right\rangle$ (e.g. that the wave functions of both particles will be found to propagate in certain directions) we have to take the squared modulus of the inner product of $\left|p^{+} e^{+}, \psi_{f}\right\rangle$ and the evolved state $\mid p^{+} e^{+}$, $\left.t_{1}\right\rangle$. Using this recipe, we can arrive at the following two formulas representing the probabilities of detecting particles scattered in appropriate directions $A$ and $B$ :

$$
\begin{gathered}
\text { (1) } \operatorname{Pr}\left(\left|p^{+}, \psi_{L}\right\rangle \otimes\left|e^{+}, \psi_{R}\right\rangle \rightsquigarrow\left|p^{+}, \psi_{A}\right\rangle \otimes\left|e^{+}, \psi_{B}\right\rangle\right) \\
=\left|\left\langle p^{+}, \psi_{A}\left|\otimes\left\langle e^{+}, \psi_{B}|U(t)| p^{+}, \psi_{L}\right\rangle \otimes\right| e^{+}, \psi_{R}\right\rangle\right|^{2} \\
\text { (2) } \operatorname{Pr}\left(\left|p^{+}, \psi_{L}\right\rangle \otimes\left|e^{+}, \psi_{R}\right\rangle \rightsquigarrow\left|p^{+}, \psi_{B}\right\rangle \otimes\left|e^{+}, \psi_{A}\right\rangle\right) \\
=\left|\left\langle p^{+}, \psi_{B}\left|\otimes\left\langle e^{+}, \psi_{A}|U(t)| p^{+}, \psi_{L}\right\rangle \otimes\right| e^{+}, \psi_{R}\right\rangle\right|^{2} .
\end{gathered}
$$

Formula (1) gives the numerical value for the probability of the proton being detected in state $\psi_{A}$ and the positron in state $\psi_{B}$, while formula (2) expresses the probability of the detection of the positron in $\psi_{A}$ and the proton in $\psi_{B}$. Numbers $\left\langle p^{+}, \psi_{A}\right| \otimes$ $\left\langle e^{+}, \psi_{B}|U(t)| p^{+}, \psi_{L}\right\rangle \otimes\left|e^{+}, \psi_{R}\right\rangle$ and $\left\langle p^{+}, \psi_{B}\left|\otimes\left\langle e^{+}, \psi_{A}|U(t)| p^{+}, \psi_{L}\right\rangle \otimes\right| e^{+}, \psi_{R}\right\rangle$ are called 
transition (or probability) amplitudes corresponding, respectively, to the transition of the proton from its initial state $\psi_{L}$ to the final state $\psi_{A}$ and the positron from $\psi_{R}$ to $\psi_{B}$, and to the transition of the proton from $\psi_{L}$ to $\psi_{B}$ and the positron from $\psi_{R}$ to $\psi_{A}$. ${ }^{9}$ If we are only interested in estimating the probability that one particle will be scattered in the left-down direction $A$ while the other particle goes in the opposite, right-up direction $B$ (without telling which one is the proton and which one the positron) all we have to do is add the probabilities (1) and (2) in an entirely classical fashion:

$$
\begin{aligned}
\operatorname{Pr}\left(\psi_{L}, \psi_{R} \leadsto \psi_{A}, \psi_{B}\right)= & \left|\left\langle p^{+}, \psi_{A}\left|\otimes\left\langle e^{+}, \psi_{B}|U(t)| p^{+}, \psi_{L}\right\rangle \otimes\right| e^{+}, \psi_{R}\right\rangle\right|^{2} \\
& +\left|\left\langle p^{+}, \psi_{B}\left|\otimes\left\langle e^{+}, \psi_{A}|U(t)| p^{+}, \psi_{L}\right\rangle \otimes\right| e^{+}, \psi_{R}\right\rangle\right|^{2} .
\end{aligned}
$$

So far we have encountered no significant conceptual difficulties with the issue of diachronic identifications of particles involved in the scattering process. Each particle recorded in state $\psi_{A}$ or $\psi_{B}$ after the interaction can be traced back to one of the two particles before the interaction, thanks to the distinguishing state-independent properties. The reason we have gone through this simple exercise is to prepare the formalism for the task of an interpretation of scattering processes in the not-so-straightforward cases of indistinguishable particles. That way we will be able to use arguments from formal analogy (or disanalogy) with the simple case in order to derive plausible interpretations of more controversial cases.

\section{The case of indistinguishable particles}

The second considered case will be that of scattering two indistinguishable particles, e.g. two protons. The initial situation will be identical as in the previous case: one proton is coming from the left while the other approaches from the right. However, formally we can no longer describe the initial state of the system as $\mid p^{+}$, $\left.\psi_{L}\right\rangle_{1} \otimes\left|p^{+}, \psi_{R}\right\rangle_{2}$. The reason for this is that protons are fermions, and therefore their joint state has to be antisymmetric. So the pre-interaction state must be written as follows:

$$
\text { (4) } \frac{1}{\sqrt{2}}\left(\left|p^{+}, \psi_{L}\right\rangle_{1} \otimes\left|p^{+}, \psi_{R}\right\rangle_{2}-\left|p^{+}, \psi_{R}\right\rangle_{1} \otimes\left|p^{+}, \psi_{L}\right\rangle_{2}\right)
$$

Similar restrictions apply to the final state, whose transition probability we are trying to calculate. That is, rather than distinguishing two possible realizations of the final state

\footnotetext{
${ }^{9}$ Note that the transition amplitudes expressed with the help of the initial and final states must contain the unitary evolution operator $U(t)$ which transforms the initial state as a result of the interaction between the particles. The final state, on the other hand, is the state recorded in the experiment (detection of particles as scattered at a certain angle). In addition, we may observe that if operator $U(t)$ factorizes (i.e. $U(t)=U_{p}(t) \otimes$ $U_{e}(t)$ ), then the above transition amplitudes can be presented in a simpler product form: $\left\langle p^{+}, \psi_{A}\left|U_{p}(t)\right| p^{+}, \psi_{L}\right\rangle$ $\left\langle e^{+}, \psi_{B}\left|U_{e}(t)\right| e^{+}, \psi_{R}\right\rangle$ and $\left\langle p^{+}, \psi_{B}\left|U_{p}(t)\right| p^{+}, \psi_{L}\right\rangle\left\langle e^{+}, \psi_{A}\left|U_{e}(t)\right| e^{+}, \psi_{R}\right\rangle$. See (Sakurai \& Napolitano 2011, pp. 86-89) for an elementary explanation of the notion of transition amplitudes.
} 
in the form of product states $\left|p^{+}, \psi_{A}\right\rangle_{1} \otimes\left|p^{+}, \psi_{B}\right\rangle_{2}$ and $\left|p^{+}, \psi_{B}\right\rangle_{1} \otimes\left|p^{+}, \psi_{A}\right\rangle_{2}$, we have to take the antisymmetric version as the only available state after the interaction:

$$
\text { (5) } \frac{1}{\sqrt{2}}\left(\left|p^{+}, \psi_{A}\right\rangle_{1} \otimes\left|p^{+}, \psi_{B}\right\rangle_{2}-\left|p^{+}, \psi_{B}\right\rangle_{1} \otimes\left|p^{+}, \psi_{A}\right\rangle_{2}\right)
$$

Given that the unitary evolution operator $U(t)$ must be symmetric, we can again calculate the probability of finding the protons scattered in the selected directions by taking the inner product of the final state and the evolved state, and then squaring its modulus. The result of the straightforward calculation will be as follows (thanks to the symmetry of $U(t)$ we can apply the equalities $\left\langle p^{+}, \psi_{A}\left|\otimes\left\langle p^{+}, \psi_{B}|U(t)| p^{+}, \psi_{L}\right\rangle \otimes\right| p^{+}, \psi_{R}\right\rangle=$ $\left\langle p^{+}, \psi_{B}\left|\otimes\left\langle p^{+}, \psi_{A}|U(t)| p^{+}, \psi_{R}\right\rangle \otimes\right| p^{+}, \psi_{L}\right\rangle$ and $\left\langle p^{+}, \psi_{A}\left|\otimes\left\langle p^{+}, \psi_{B}|U(t)| p^{+}, \psi_{R}\right\rangle \otimes\right| p^{+}, \psi_{L}\right\rangle=$ $\left.\left\langle p^{+}, \psi_{B}\left|\otimes\left\langle p^{+}, \psi_{A}|U(t)| p^{+}, \psi_{L}\right\rangle \otimes\right| p^{+}, \psi_{R}\right\rangle\right):$

$$
\begin{gathered}
\text { (6) } \operatorname{Pr}\left(\psi_{L}, \psi_{R} \leadsto \psi_{A}, \psi_{B}\right)=\mid\left\langle p^{+}, \psi_{A}\left|\otimes\left\langle p^{+}, \psi_{B}|U(t)| p^{+}, \psi_{L}\right\rangle \otimes\right| p^{+}, \psi_{R}\right\rangle \\
-\left.\left\langle p^{+}, \psi_{B}\left|\otimes\left\langle p^{+}, \psi_{A}|U(t)| p^{+}, \psi_{L}\right\rangle \otimes\right| p^{+}, \psi_{R}\right\rangle\right|^{2} .
\end{gathered}
$$

Observe first that in the current case the formalism does not even allow us to consider the probabilities of obtaining more specific transitions $\psi_{L} \leadsto \psi_{A} ; \psi_{R} \leadsto \psi_{B}$ and $\psi_{L} \leadsto \psi_{B}$; $\psi_{L} \leadsto \psi_{A}$, as given by formulas (1) and (2). The reason is the unphysicality of the product states $\left|p^{+}, \psi_{A}\right\rangle_{1} \otimes\left|p^{+}, \psi_{B}\right\rangle_{2}$ and $\left|p^{+}, \psi_{B}\right\rangle_{1} \otimes\left|p^{+}, \psi_{A}\right\rangle_{2}$ invoked above. The case in which proton number 1 ends up left-down and proton number 2 ends up right-up is physically indistinguishable from the case in which the protons are swapped. So the only way to calculate the total probability $\operatorname{Pr}\left(\psi_{L}, \psi_{R} \rightarrow \psi_{A}, \psi_{B}\right)$ is to take the initial and final states in their proper antisymmetric forms, rather than summing individual probabilities $\operatorname{Pr}\left(\psi_{L} \rightsquigarrow \psi_{A}, \psi_{R} \rightsquigarrow \psi_{B}\right)$ and $\operatorname{Pr}\left(\psi_{L} \rightsquigarrow \psi_{B}, \psi_{R} \rightsquigarrow \psi_{A}\right)$. As a result, formula (6) differs from its distinguishable-case counterpart (3) in one important way: while (3) is the sum of squared amplitudes, (6) is equal to the sum of squared amplitudes minus an additional component. This so-called interference component shows in real experiments in the form of the well-known interference pattern (the probability of finding the scattered particles in different directions varies in a regular way with the angle). Thus by measuring the probability distribution over the possible angles at which the particles can scatter we can empirically differentiate between the cases of distinguishable and indistinguishable particles. Only indistinguishable particles can interact in such a way that their wave functions will interfere with one another.

In order to derive from this case some metaphysical consequences regarding the fate of the diachronic identity of particles, we have to choose which approach to synchronic individuation to follow: the orthodox or heterodox one. Starting with the first option, we should recall that the orthodoxy treats labels 1 and 2 present in the mathematical representations of the initial and final states (4) and (5) as referential. This means that the questions of the diachronic identity between the pre- and post-interactions particles can be cast with the help of the labels as follows: "Is particle 1 occupying state (5) at $t_{2}$ identical with particle 1 or particle 2 occupying state (4) at $t_{1}$ ?", and similarly for particle number 2 . And it should be clear that, given the qualitative indiscernibility of particles labeled 1 and 2 before and after the interaction, no physical reason can be given for any specific diachronic identification. Any conceivable argument in favor, 
let's say, of the diachronic identification between the particle labeled as "1" in (5) and the particle labeled as "1" in (4), can be turned, mutatis mutandis, into an argument in favor of the alternative identification of particle 1 in (5) and particle 2 in (4) - this is a simple consequence of the permutation invariance of the states (4) and (5). (This argument of course presupposes that all physical facts regarding the system of two protons at times $t_{1}$ and $t_{2}$ are exhausted by their initial and final physical states. If we allowed into the picture some extraneous elements not encompassed in the quantummechanical states, for instance Bohmian trajectories, the result would be entirely different.)

Thus under the orthodox interpretation the issue of the diachronic identification of particles of the same type cannot be settled by physics. And this consequence holds regardless of the physical details of the interaction between the considered particles. Even if we assumed that the initial state (4) of the two electrons does not change in time, still at every possible moment of the temporal existence of the particles occupying this state it is not settled by the physics whether it is particle 1 or 2 that is a continuant of particle 1 at $t_{1}$. Asking questions of that type would be as futile as asking whether a dollar that I deposited one day into my bank account is numerically identical with the dollar that I withdrew the next day. On the other hand, we have to admit that nothing in the physics can prevent an obstinate proponent of diachronic identity from stubbornly insisting that there is some non-empirical thread of identity that connects the two indiscernible particles at each moment of their existence. This position is extremely unintuitive (it acknowledges the existence of 'brute' facts of numerical identity that can never be known by us), and it clearly commits us to some variant of haecceitism, or the existence of primitive identities, but there is no knock-down argument that could eliminate it as a logically possible option.

When we adopt the heterodox approach to the problem of individualization of particles, the situation presents itself in a slightly different light. According to this method of individuation, when the protons are in state (4), it is true that one proton occupies state $\left|\psi_{L}\right\rangle$, while the other occupies $\left|\psi_{R}\right\rangle$. Thus we can talk about the $L$-proton and the $R$-proton before the interaction. Similarly, we can use states $\left|\psi_{A}\right\rangle$ and $\left|\psi_{B}\right\rangle$ in order to differentiate between the protons after the interaction (and the subsequent detection). But the issue of how to connect these particles diachronically remains open to debate. Can there be any physical reason to claim that the $A$-proton at time $t_{2}$ is for instance identical with the $L$-proton at $t_{1}$ ? Nothing in the experimental situation can tell us whether the particle detected as spreading in the $A$ direction came from the left or the right. But can't we again apply the distinction between what can be known and what is? Perhaps the $A$-particle $i s$ identical with the $L$-particle (or the $R$-particle), only we can't know which is the case. Apart from the fact that, as before, this position commits us to primitive identities (or haecceities), there is one more problem with such a solution that has to do with the physical details of the scattering scenario considered above. If in fact the $A$-particle is identical either with the $L$-particle or the $R$-particle, then why can't we calculate the probabilities of these two scenarios separately, and then add them classically, exactly as in the case of distinguishable particles? But that way we would end up with a formula that looks like (3) rather than (6), and we know that this is empirically incorrect (the interference effects implied by (6) and not by (3) are empirically observable when we scatter particles of the same type). So it looks like 
the hypothesis that the particles retain their diachronic identities can be put to the test, and the test speaks unambiguously against adopting this hypothesis.

Admittedly, this conclusion can be resisted. It may be claimed that the fact that we can't calculate transition probabilities for the scenarios $\psi_{L} \leadsto \psi_{A}, \psi_{R} \leadsto$ $\psi_{B}$ and $\psi_{L} \leadsto \psi_{B}, \psi_{R} \rightsquigarrow \psi_{A}$ taken separately, does not indicate that the particles lose their diachronic identities, but is merely a result of the formal restrictions on the available states of fermions. As we have already stressed, the initial and final states can never take the form of product states due to the requirement of symmetry. Thus it is formally impossible to derive probabilities $\operatorname{Pr}\left(\psi_{L} \leadsto \psi_{A}, \psi_{R}\right.$ $\left.\leadsto \psi_{B}\right)$ and $\operatorname{Pr}\left(\psi_{L} \rightsquigarrow \psi_{B}, \psi_{R} \rightsquigarrow \psi_{A}\right)$, as given in formulas (1) and (2), since this requires that there be two distinct product states in which the system may end up after the interaction (and that the initial state be a product state, too). Consequently, the only way to arrive at the formula characterizing the total probability $\operatorname{Pr}\left(\psi_{L}, \psi_{R} \leadsto \psi_{A}, \psi_{B}\right)$ is through the direct calculation using the antisymmetric initial and final state. But this is merely a consequence of the adopted formalism, and not a sign of the breakage of the diachronic identities of the particles.

In response we may observe that while certainly formula (6) is derivable from the formal requirement of antisymmetrization, this fact should not prevent us from asking further questions regarding an interpretation of the derived mathematical expression for the probability $\operatorname{Pr}\left(\psi_{L}, \psi_{R} \rightsquigarrow \psi_{A}, \psi_{B}\right)$. Comparing (6) with (3) we can clearly recognize in both expressions the amplitudes of the general form $\left\langle\psi_{A}\left|\otimes\left\langle\psi_{B}|U(t)| \psi_{L}\right\rangle \otimes\right| \psi_{R}\right\rangle$ and $\left\langle\psi_{B}\left|\otimes\left\langle\psi_{A}|U(t)| \psi_{L}\right\rangle \otimes\right| \psi_{R}\right\rangle$, which in the case of distinguishable particles represented the probabilities of specific transitions $\psi_{L} \leadsto \psi_{A}, \psi_{R} \rightsquigarrow \psi_{B}$ and $\psi_{L} \leadsto \psi_{B}, \psi_{R} \leadsto \psi_{A}$. Since the amplitudes in expression (6) are combined in a different manner than in (3), the result is that the total probability is not the sum of the individual probabilities of the above specific transitions. This gives us good (albeit perhaps not conclusive) reasons to believe that the cases of scatterings of indistinguishable particles cannot be divided up into two subcategories depending on which particle ended up where. Taking this plausibility argument into account, I will continue to treat the existence of the interference pattern implied by formula (6) as a symptom of the loss of the diachronic identity between the particles identified before and after the interaction.

To sum up, both approaches to quantum individuality lead to the same general conclusion regarding diachronic identity in scattering experiments of the sort described above. The conclusion is that in the scenario involving two indistinguishable particles whose states are represented by their wave functions while ignoring spin, it is in principle impossible to identify particles after the interactions with appropriate particles before the interaction. However, in each approach the arguments in favor of this joint conclusion were different. This difference will turn out to be rather important in the case we will consider next.

\section{The case of indistinguishable particles with differentiating properties}

The scenario discussed in this section again involves two indistinguishable particles (e.g. two protons). But this time we will assume that in addition to the spatial degrees of freedom, the particles are also characterized by some internal degrees of freedom, for 
instance spin (intrinsic angular momentum). Suppose, for example, that the incoming particles are characterized by some definite and opposite values of spin in a certain direction that we will symbolize as $\uparrow$ and $\downarrow$. Assuming that the particle coming from the left is the one that has spin "up" (see Fig. 2), we can write the initial state of both particles before the interaction as follows ${ }^{10}$ :

$$
\text { (7) } \frac{1}{\sqrt{2}}\left(\left|\uparrow, \psi_{L}\right\rangle_{1} \otimes\left|\downarrow, \psi_{R}\right\rangle_{2}-\left|\downarrow, \psi_{R}\right\rangle_{1} \otimes\left|\uparrow, \psi_{L}\right\rangle_{2}\right)
$$

As before, we are ultimately interested in calculating the probability $\operatorname{Pr}\left(\psi_{L}, \psi_{R} \leadsto \psi_{A}\right.$, $\psi_{B}$ ) of detecting the protons scattered in particular directions $A$ and $B$. But given that spin and position are independent (and commuting) observables, we have to consider two possible realizations of the final state that lead to the same observable effect, where one possibility is that the particle detected in $A$ has spin up while the other, $B$-particle has spin down, and the other possibility reverses this combination ( $A$-particle with spin down, and $B$-particle with spin up). We don't have to take into account the two remaining options (both particles having spins up or spins down), since the total spin has to be preserved, so the final state has to be one of the following:

$$
\begin{aligned}
& \text { (8) } \frac{1}{\sqrt{2}}\left(\left|\uparrow, \psi_{A}\right\rangle_{1} \otimes\left|\downarrow, \psi_{B}\right\rangle_{2}-\left|\downarrow, \psi_{B}\right\rangle_{1} \otimes\left|\uparrow, \psi_{A}\right\rangle_{2}\right) \\
& \text { (9) } \frac{1}{\sqrt{2}}\left(\left|\downarrow, \psi_{A}\right\rangle_{1} \otimes\left|\uparrow, \psi_{B}\right\rangle_{2}-\left|\uparrow, \psi_{B}\right\rangle_{1} \otimes\left|\downarrow, \psi_{A}\right\rangle_{2}\right)
\end{aligned}
$$

In order to calculate the transition amplitudes between state (7) on the one hand and state (8) or (9) on the other, we will make one crucial assumption - namely, that the interaction between the particles does not affect spin. In other words, the evolution operator in the spin space is just the identity operator. Given this assumption, the corresponding probabilities will be as follows:

$$
\operatorname{Pr}\left(\uparrow \psi_{L}, \downarrow \psi_{R}^{\rightsquigarrow \uparrow} \uparrow \psi_{A}, \downarrow \psi_{B}\right)=\left|\left\langle\psi_{A}\left|\otimes\left\langle\psi_{B}|U(t)| \psi_{L}\right\rangle \otimes\right| \psi_{R}\right\rangle\right|^{2}
$$

(11) $\operatorname{Pr}\left(\uparrow \psi_{L}, \downarrow \psi_{R} \rightsquigarrow \downarrow \psi_{A}, \uparrow \psi_{B}\right)=\left|\left\langle\psi_{B}\left|\otimes\left\langle\psi_{A}|U(t)| \psi_{L}\right\rangle \otimes\right| \psi_{R}\right\rangle\right|^{2}$

In deriving the above expressions, we have made use of the orthonormality relation between spin-states $|\uparrow\rangle$ and $|\downarrow\rangle$. Interestingly, the resulting formulas are mathematically identical to the ones obtained in the case of distinguishable particles (formulas (1) and

\footnotetext{
$\overline{{ }^{10}}$ To avoid clutter I omit the symbol " $p$ " " used earlier to indicate that appropriate states are attributed to protons. 

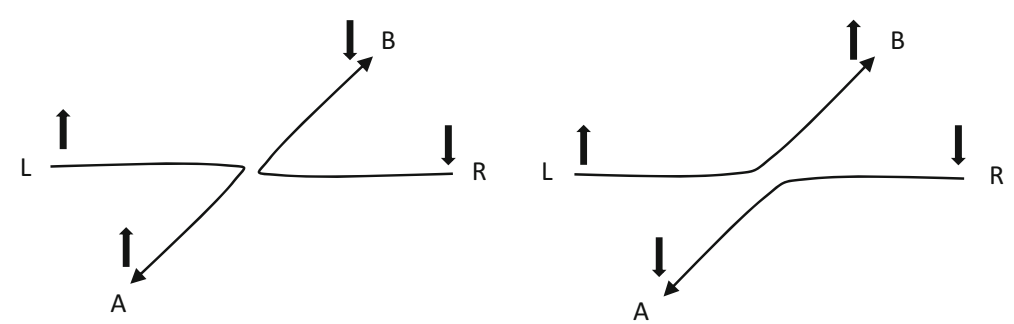

Fig. 2 Scattering with spins

(2)). And so is the formula for the total probability, in which we simply add the probabilities of the two alternative scenarios:

$$
\text { (12) } \begin{aligned}
\operatorname{Pr}\left(\psi_{L}, \psi_{R} \rightsquigarrow \psi_{A}, \psi_{B}\right)= & \left|\left\langle\psi_{A}\left|\otimes\left\langle\psi_{B}|U(t)| \psi_{L}\right\rangle \otimes\right| \psi_{R}\right\rangle\right|^{2} \\
& +\left|\left\langle\psi_{B}\left|\otimes\left\langle\psi_{A}|U(t)| \psi_{L}\right\rangle \otimes\right| \psi_{R}\right\rangle\right|^{2} .
\end{aligned}
$$

On the basis of the formal analogy between formulas (10), (11), (12) and (1), (2), (3), we may be tempted to conclude that in the currently considered case of indistinguishable particles with spin the relation of diachronic identity holds between appropriate particles before and after interactions. However, let us examine closer this conjecture using the two available interpretations of quantum individuation. As can be easily verified, the heterodox approach fully confirms the hypothesis that in the aboveconsidered case particles retain their identities over time. States (7), (8) and (9) are obtainable from the appropriate product states through the operation of antisymmetrization, and therefore, according to the heterodox approach, it is legitimate to attribute to individual particles the properties corresponding to these factorizable states. Thus in the initial state (7) we say that there is one particle whose wave function is coming from $L$ and whose spin is up, and one particle coming from $R$ with spin down. Similarly, the final state (8) is the state of a system of two particles, one of which is propagating towards $A$ with spin up, and the other is entering $B$ and possesses spin down. The alternative state (9) merely reverses the spins of the particles moving in the directions of $A$ and $B$.

Given that the spin of the scattering particles is assumed to be preserved during the interaction, it stands to reason to make the following identifications. When the final state of the system happens to be (8), the outgoing particle detected in $A$ should be identified with the incoming particle from the left (since they both possess the same spin up), and correspondingly, the particle detected in $B$ ought to be identified with the $R$-particle. The state (9) switches these identifications. ${ }^{11}$ Now, if the experimental setting does not make it possible to measure the spins of the outgoing particles, only the directions in which they are scattered, we may not be able to tell which of the detected particles is identical with which incoming proton. But this is a mere epistemological issue, not an ontological one. Each detected particle possesses its spin which uniquely identifies it with one of the incoming particles. Thus the total probability (12)

\footnotetext{
${ }^{11}$ The possibility of making unique identifications of same-type particles with the help of conserved quantities (e.g. spin in our example) is mentioned by Nick Huggett and Tom Imbo (2009, p. 315). They call this, perhaps slightly confusingly, "approximate distinguishability".
} 
is the sum of the two probabilities corresponding to the two possible values of spin, and in consequence there is no interference effect. The heterodox approach is thus consistent with the assumption that the lack or presence of the interference effects is a criterion of the existence or non-existence of the diachronic identity connecting the particles before and after the interaction.

Not so with the second, orthodox interpretation. The keystone of this approach is the assumption that the labels used in the antisymmetric states of groups of fermions refer to individual particles. Thus the question of the diachronic identification reduces to the question of whether the particle bearing label 1 in the post-interaction state (8) or (9) is identical with the particle 1 or 2 in the pre-interaction state (7). And it is clear that the argument presented in the previous section applies to this case as well: due to the permutation-invariance of all involved states, no specific identification can be supported by any physical facts or data. The fact that spin is preserved in the interaction is irrelevant, since, strictly speaking, there is no particle that possesses any definite spin before or after the interaction. Particle 1 occupying state (7) is actually in a mixed state with respect to both position and spin, i.e. it is equally probable that its spin is up or down (and that it is coming from $L$ or $R$ ). The possibility of diachronic identification is excluded from the outset, irrespective of what type of evolution both particles undergo. Consequently, the interference effects have nothing to do with the existence of diachronic identity between the particles participating in the scattering process. Regardless of whether the interference pattern is present or not, quantum particles of the same type are never identifiable over time according to the position we call orthodoxy.

\section{Conclusion: How to keep track of quantum objects}

I would like to draw two general morals from the considered case. The first one concerns the ongoing debate between the orthodox and heterodox approaches to quantum individuation. They present us with two incompatible and irreconcilable conceptions of quantum objects, and the issue of which one to choose becomes rather pressing in the foundational discussions on quantum mechanics. The orthodoxy, based on the 'literal' interpretation of labels, tends to treat groups of particles of the same type not as collections of individuals, but rather as aggregates of entirely indiscernible entities. Each electron in the universe is characterized by exactly the same reduced state, and consequently cannot be individuated from the rest of the electrons. Alternatively, the orthodox interpretation can be coupled with the rather controversial assumption that each quantum object possesses its unique identity in the form of a nonqualitative, un-empirical primitive 'thisness' (haecceity), whose linguistic representation is given in the form of individual constants (labels) rather than qualitative predicates. On the other hand, the heterodox proposal rehabilitates to a certain extent the conception of objects individuated by their qualitative, empirical properties, in accordance with the principle of the identity of indiscernibles. This is made possible thanks to the assumption that the way to refer to individual particles is not through labels but through symmetric 'discernibility' projection operators (like the one given in ft. 7) acting in the $\mathrm{N}$-fold tensor product of Hilbert spaces. Consequently, it can be proven that fermions, which have to occupy antisymmetric states, can always be 
discerned and individuated by some non-trivial properties, while bosons may or may not be discernible, depending on what particular symmetric states they occupy.

I believe that the examples involving the comparisons of diachronic identity in scattering experiments discussed above can be used as a measuring rod to evaluate both approaches to quantum individuation. The fundamental fact to keep in mind is that there are two ways in which particles of the same type can interact with each other. If the interaction does not admit any conserved quantity that can be used to connect postand pre-interaction particles, the scattering will display a clear interference pattern. On the other hand, the presence of a qualitative 'mark', for instance in the form of a specific value of spin component that is not affected by the interaction, changes the quantum-mechanical predictions regarding the probability amplitudes in such a way that the total probability becomes the simple sum of the partial probabilities, and the interference pattern vanishes. These facts, predicted theoretically and verifiable empirically, are easily accounted for by the heterodox approach. Under this approach, the difference between the two cases is explained by reference to the existence or nonexistence of the facts of diachronic identity between particles before and after the interaction. That is, the interference effects are correlated with, and accounted for by, the in-principle impossibility to tell which of the detected particles is identical with which of the incoming particles. On the other hand, the loss of interference effects is explainable by the fact that the post-interaction particles, individuated synchronically by their distinct properties (e.g. position and spin), can be unambiguously connected with the pre-interaction particles via their conserved property - spin.

In contrast to that, the orthodox approach is unable to give a deeper explanation for the difference between the interference and non-interference variants of the scattering experiments. In both cases the diachronic identifications are excluded from the outset, due to the fact that in this approach it is labels and not qualitative properties that individuate synchronically the interacting particles. Thus the different observable effects in variants of scattering experiments with and without differentiating marks are treated as brute facts that follow from the quantum-mechanical formalism but have no deeper meaning. Moreover, the orthodox approach has to abandon the intuitive interpretations of some parts of the formalism that are naturally admitted under the alternative approach. This applies primarily to the separate transition amplitudes present in formulas (1), (2), (3), (6), (10), (11) and (12). For instance, a natural interpretation of the amplitude $\left\langle\psi_{B} \otimes\left\langle\psi_{A}|U(t)| \psi_{L}\right\rangle \otimes \mid \psi_{R}\right\rangle$ is that it refers to the process in which the particle that initially occupied state $\left|\psi_{L}\right\rangle$ is now detected to be in state $\left|\psi_{B}\right\rangle$ (while simultaneously the particle recorded in $\left|\psi_{A}\right\rangle$ turns out to be the one that came from the state $\left.\left|\psi_{R}\right\rangle\right)$. This interpretation of transition amplitudes lies at the heart of the argument that the process whose total probability is given as in formula (6) cannot be considered as 'consisting' of two channels connected by classical disjunction. But for the proponent of the orthodox conception the transition amplitudes of the form $\mid\left\langle\psi_{B} \otimes\right.$ $\left\langle\psi_{A}|U(t)| \psi_{L}\right\rangle \otimes\left|\psi_{R}\right\rangle$ are essentially meaningless as a means to refer to real physical processes involving quantum objects persisting in time. This is so, because the amplitudes do not even contain labels, and only labels offer a way to refer to individual particles according to the orthodoxy. The labels get 'washed away' when we calculate the inner products of the initial and final states (4) and (5) or (7), (8) and (9). Mathematically, we are moving here from the product of labeled Hilbert spaces to the simple space of complex numbers that do not wear any identifiable labels on their 
sleeves. While certainly not decisive, I see the above arguments as seriously undermining the orthodox approach. The heterodox conception, on the other hand, provides us with straightforward interpretations of the parts of the formalism that are used to calculate the total probabilities. In addition to that, it squares nicely with the way physicists describe the experimental settings of the scattering experiments in terms of left- and right-particle (see e.g. (Feynman et al. 1965, chapters 3 and 4)).

It may be objected that the foregoing argument is based on the unrealistic assumption that in the spin-scattering example the spins of the particles are indeed not affected by the mutual interactions. ${ }^{12}$ This assumption may be questioned on the basis of the fact that spins easily couple with electromagnetic fields and thus are unlikely to remain unchanged when the charged particles interact. This apparently blunts the force of the argument against the orthodox approach to individuation. Now, I am neither prepared nor willing to discuss here the physics of the interactions between spins and electromagnetic fields, noting only in passing that Richard Feynman in his famous lectures preempts this objection by pointing out that "[i]f the energy of the experiment is low enough, the magnetic forces due to the currents will be small and the spins will not be affected" (Feynman et al. 1965, chap. 3). However, regardless of the (un)physicality of the assumption of the spin preservation, I believe that the argument is strong enough to be useful in the debate between orthodoxy and heterodoxy. The key point is that the orthodox approach to individuality is unable to give a deeper explanation of the possibility (however remote) of the non-interference inducing interactions between same-type particles. It does not matter much how narrow these cases are - as long as they represent a theoretical possibility, it is better to distinguish them from the cases in which the scattering gives rise to interference effects. The heterodoxy naturally explains this difference in terms of the existence or non-existence of the diachronic identity between particles, while the orthodoxy must treat it as a brute fact, since diachronic identity is not available to it in any circumstances.

The second moral I will try to extract now concerns the specific conception of quantum particles that can be derived from the heterodox interpretation. It may be tempting to think that the heterodox approach reinstates the classical way of describing elementary particles by effectively ('for all practical purposes') treating the antisymmetric, permutation-invariant states of the form (4) or (5) as if they were ordinary product states. But this would be an entirely incorrect conclusion. For starters, the formal derivations of the transition amplitudes in scattering experiments require that we use the initial and final states in their full antisymmetric forms, otherwise the results would be widely off mark. But even more important from the philosophical point of view is the fact that particles which are synchronically individuated by their properties may lose their identity in the course of their temporal evolution.

Compare this with the classical, naïve view of physical objects. There are two intuitive and independent principles, deeply rooted in our pre-philosophical thoughts, that shape our ordinary, common concept of a material thing. The first principle is the already mentioned identity of the indiscernibles (PII), or, as it is sometimes called, the distinguishability of the distinct. We have a strong pre-theoretical intuition that each individual material object, be it a snowflake, a drop of water, or a neutron star, has some unique features that differentiate it from the rest of apparently similar objects

$\overline{12}$ Thanks to an anonymous referee for raising this objection. 
(other snowflakes, drops of water or neutron stars). But the second principle concerns the identity of objects in time. It stipulates that it always makes sense to ask whether an object selected at a particular moment is identical (or not) with another, similar object taken at a different time, and that such a question should always have a unique answer, regardless of whether we may ever know it. An actual identification of complex physical things over time may present us with a serious epistemological problem, as evidenced by the famous case of the ship of Theseus. However, putting aside the unquestionably serious issue of the vagueness of diachronic identity, the predominant intuition is that objects - especially fundamental ones - keep their unique and unambiguous identity over time as long as they exist. ${ }^{13}$ From this it follows that if we have a pair of distinct physical objects taken at two different moments, and if no object in the pair has been destroyed or created in the intervening moments, then it is objectively determined which entity at one moment is identical with which entity at the other moment, regardless of our ability to learn which is the case.

The main focus of the recent discussions regarding the concept of quantum objects has been on the first of the two intuitive principles, i.e. the synchronic PII. With respect to that problem, the heterodox approach to individuality is surprisingly classical, i.e. it rehabilitates the notion of qualitative discernibility by physical properties in the majority of interesting cases. However, the classicality of the heterodoxy goes only that far. The non-classical, unintuitive features of the quantum-mechanical description take center stage when we consider how physical systems evolve in time. The 'paradox' of diachronic identity, as present in the second of the above-considered cases of particle scattering, can be concisely presented as follows. There is a complex system consisting of two particles of which the following three theses seem to be true:

(i) The total system at time $t_{2}$ is identical with the total system at $t_{1}$ (no particles are created or destroyed in the process).

(ii) At both times $t_{1}$ and $t_{2}$ there are two distinct particles that are individuated by some of their properties.

(iii) No particle selected at $t_{2}$ is identical with any particle at $t_{1}$.

This looks like a logical inconsistency, but only if we adopt the second intuitive principle discussed in the previous paragraph. That is, without the assumption that the facts of diachronic identity are objectively determined, we may try to avoid the logical difficulty by pointing out that each individual particle at later time $t_{2}$ is somehow genetically linked to both particles at time $t_{1}$ without being identical with any of them. A proper analysis of this supposition requires of course some further work on a notion of identity over time which could accommodate the facts of multiple genetic connections weaker than identity. ${ }^{14}$ Perhaps we should even abandon one presupposition of the current analysis, spelled out at the beginning of the paper, that diachronic identity is just numerical identity taken at different temporal points. However, I would like to leave a thorough analysis of this option for another occasion.

\footnotetext{
${ }^{13}$ Simon Saunders for instance insists that for an individual it should be true by definition that it "can be uniquely identified throughout the time it persists" (Saunders 2015, p. 165).

14 There are some interesting analogies worth exploring between the quantum case considered here and the case of split brains and failure of personal identity so famously discussed in (Parfit 1971).
} 
Acknowledgments I am grateful to two anonymous referees for their comments to an earlier version of this paper. The work on this paper was supported by grant No. 2017/25/B/HS1/00620 from the National Science Centre, Poland.

Open Access This article is licensed under a Creative Commons Attribution 4.0 International License, which permits use, sharing, adaptation, distribution and reproduction in any medium or format, as long as you give appropriate credit to the original author(s) and the source, provide a link to the Creative Commons licence, and indicate if changes were made. The images or other third party material in this article are included in the article's Creative Commons licence, unless indicated otherwise in a credit line to the material. If material is not included in the article's Creative Commons licence and your intended use is not permitted by statutory regulation or exceeds the permitted use, you will need to obtain permission directly from the copyright holder. To view a copy of this licence, visit http://creativecommons.org/licenses/by/4.0/.

\section{Bibliography}

Bigaj, T. (2015), "Exchanging quantum particles”, in: P.E. Bour, G. Heinzmann, W. Hodges, \& P. SchroederHeister (eds.), 14th CLMPS 2011 proceedings, Philosophia scientiae 19, 185-198.

Bigaj, T. (2016). On some troubles with the metaphysics of fermionic compositions. Foundations of Physics, 46(9), 1168-1184.

Bigaj, T. (2020a). On discernibility in symmetric languages: The case of quantum particles. Synthese. https://doi.org/10.1007/s11229-020-02582-7.

Bigaj, T. (2020b), "How to justify the symmetrization postulate in quantum mechanics", forthcoming in Journal for General Philosophy of Science.

Butterfield, J. (1993). Interpretation and identity in quantum theory. Studies in History and Philosophy of Science, 24, 443-476.

Caulton, A. (2014), "Qualitative individuation in permutation-invariant quantum mechanics", arXive: $1409.0247 \mathrm{v} 1$ [quant-ph].

Caulton, A. (2015), "Is mereology empirical? Composition for fermions", in. Bigaj, T. and Wüthrich, C. (eds.) Metaphysics in Contemporary Physics, Poznan Studies in the Philosophy of the Sciences and the Humanities, Brill/Rodopi, 293-321.

Cohen-Tannoudji, C., Diu, B., and Laloë, F. (1978) Quantum Mechanics vol. 2, Wiley.

Dieks, D., \& Lubberdink, A. (2011). How classical particles emerge from the quantum world. Foundations of Physics, 41, 1051-1064.

Dieks, D. and Lubberdink, A. (2020), "Identical quantum particles as distinguishable objects", forthcoming in Journal for General Philosophy of Science.

Feynman, R., Leighton, R. and Sands, M. (eds.) (1965), The Feynman Lectures on Physics, Vol.III, Reading, Mass: Addison-Wesley, available on-line at https://www.feynmanlectures.caltech.edu/.

French, S., \& Redhead, M. (1988). Quantum physics and the identity of indiscernibles. British Journal for the Philosophy of Science, 39, 233-246.

French, S. and Rickles, D. (2003), "Understanding permutation symmetry”, in: Brading, K., and Castellani, E., eds. (2003). Symmetries in Physics: Philosophical Reflections. Cambridge University press, Cambridge, 212-238.

Friebe, C. (2014). Individuality, distinguishability and (non-)entanglement: A defense of Lebiniz's principle. Studies in History and Philosophy of Modern Physics, 48, 89-98.

Galois, A. (2016), "Identity over time", The Stanford Encyclopedia of Philosophy (winter 2016 edition), Edward N. Zalta (ed.), URL = <https://plato.stanford.edu/archives/win2016/entries /identity-time/>.

Ghirardi, G., Marinatto, L., \& Weber, T. (2002). Entanglement and properties of composite quantum systems: A conceptual and mathematical analysis. Journal of Statistical Physics, 108(112), 49-122.

Haslanger, S. (2003). Persistence through time. In M. J. Loux \& D. W. Zimmerman (Eds.), The Oxford Hanbook of metaphysics (pp. 315-354). Oxford: Oxford University Press.

Huggett, N. (2003), "Quarticles and the identity of indiscernibles", in: W K. Brading, \& E. Castellani (eds.), Symmetries in Physics, Cambridge: Cambridge University Press 239-249.

Huggett, N. and Imbo, T. (2009), "Indistinguishability", in: Friedel Weinert, Klaus Hentschel, Dan Greenberger, (eds.) Compendium of quantum physics: Concepts, Experiments, History and Philosophy, Springer-Verlag, 311-317. 
Huggett, N., \& Norton, J. (2014). Weak discernibility for quanta, the right way. British Journal for the Philosophy of Science, 65, 39-58.

Ladyman, J., Linnebo, O., \& Pettigrew, R. (2012). Identity and discernibility in philosophy and logic. The Review of Symbolic Logic, 5, 162-186.

Muller, F. A., \& Saunders, S. (2008). Discerning fermions. British Journal for the Philosophy of Science, 59, $499-548$.

Parfit, D. (1971). Personal identity. The Philosophical Review, 80(1), 3-27.

Redhead, M., \& Teller, P. (1992). Quantum physics and the identity of the indiscernibles. British Journal for the Philosophy of Science, 43, 201-218.

Sakurai, J. J., \& Napolitano, J. (2011). Modern quantum mechanics (2nd ed.). San Francisco: Adison-Wesley.

Saunders, S. (2009), "Identity of quanta" in: Friedel Weinert, Klaus Hentschel, Dan Greenberger, (eds.) Compendium of quantum physics: Concepts, Experiments, History and Philosophy, Springer-Verlag, 299-304.

Saunders, S. (2013). Indistinguishability. In R. Batterman (Ed.), Oxford handbook of philosophy of physics (pp. 340-380). Oxford: Oxford University Press.

Saunders, S. (2015). On the emergence of individuals in physics. In A. Guay \& T. Pradeau (Eds.), Individuals across sciences (pp. 165-190). Oxford: Oxford University Press.

Publisher's note Springer Nature remains neutral with regard to jurisdictional claims in published maps and institutional affiliations. 